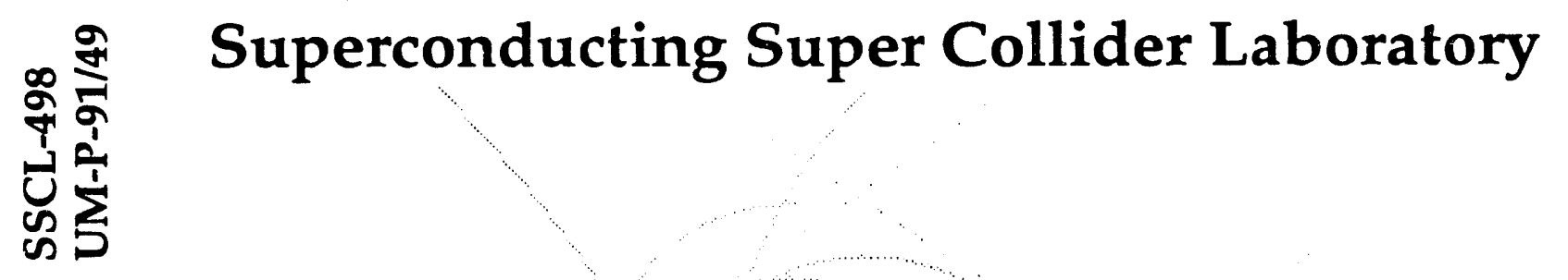

\title{
The Degeneracy of the Free Dirac Equation
}

\author{
V. Gupta, B. McKellar, and D. Wu
}




\title{
The Degeneracy of the Free Dirac Equation*
}

\author{
V. Gupta, ${ }^{1,2}$ B. H. J. McKellar, ${ }^{1}$ and D. D. $\mathrm{Wu}^{1,3,4}$ \\ ${ }^{1}$ School of Physics \\ University of Melbourne \\ Parkville, Victoria, 3052 \\ Australia \\ ${ }^{2}$ Tata Institute of Fundamental Research \\ Homi Bhabha Road, Bombay 400005, India \\ ${ }^{3}$ Institute of High Energy Physics \\ Beijing 100039 \\ P.R. China \\ ${ }^{4}$ Present Address: \\ Superconducting Super Collider Laboratory ${ }^{\dagger}$ \\ 2550 Beckleymeade Avenue \\ Dallas, Texas 75237 \\ USA \\ E-mail: DANWU@SSCVAX1.SSC.GOV
}

August 1991

* This work is supported in part by the Australia Research Council.

$\dagger$ Operated by the Universities Research Association. Inc., for the I'S. Department of Energy under Contract No. DE-AC35-89ER40486. 
$\mathrm{SSCi}-498$

UM-P-91/49

\title{
The Degeneracy of the Free Dirac Equation
}

\author{
V. Gupta, B. J. McKellar, and D. D. Wu
}

\begin{abstract}
Parity-mixed solutions of the free Dirac equation with the same 4-momentum are considered. The first-order EM energy has an electric dipole moment term whose value depends on the mixing angle. Further implications of this degeneracy to perturbative calculations are discussed. It is argued that the properties of the Dirac equation with the Coulomb potential can be used to decide the mixing angle, which should be zero.
\end{abstract}




\subsection{INTRODUCTION}

The electric dipole moment (EDM) of a fermion. either charged (e.g., the electron) or neutral (e.g., the neutron;, if found. is a special signal of $\mathrm{T}$-violation. The magnitudes or limits of the EDM of fermions discovered may put strong constraints on the viable theoretical models. The problem is that the present theoretical understanding of EDM seems very controversial. ${ }^{1}$ We feel that the controversies may be traced back to quantum electrodynamics (QED). It is commonly believed that EDM does not appear in QED, and there are different explanations about why this is so. Actually, fake EDM may appear in QED. The same fake EDM may also appear in complicated theories such as the standard $S U(3) \times S U(2) \times U(1)$ model of interactions, but in a disguised way. It might be useful to restudy $Q E D$ within this context to see how fake EDM appears and how it is eliminated.

We shall see that the degeneracy between the parity-even and -odd solutions of the free Dirac equation plays a special role in this respect. Though typical textbooks on quantum field theories take only the parity-even solutions to start perturbative calculations, it is impossible to understand EDM well without the study of parity-odd solutions as well. Since there are degenerate parity-even and -odd solutions, the results of a perturbative calculation depend on the mixing angle. if one takes the parity-mixed solutions to be the lowest-order wave functions.

A brief comparison of perturbation with degenerate lowest-order wave functions in quantum mechanics (the calculation of the Stark effect) ${ }^{2}$ will be useful. The $n=2$ free $\mathrm{H}$-atom has four degenerate wave functions, two of which are of interest: $|1\rangle=\left|2 . s, L_{z}=0\right\rangle$ and $|2\rangle=\left|2 p, L_{z}=0\right\rangle$. Linear combinations of the two are also $\mathrm{H}$-atom with the same energy:

$$
|\omega(\alpha)\rangle=\cos \alpha|1\rangle+\sin \alpha|2\rangle
$$

All these states are related by $\mathrm{O}(4)$ transformations. where $\mathrm{O}(4)$ is the degenerate group of $\mathrm{H}$-atom. When the atom is put in an external electric field $\vec{E}=E_{z} \overrightarrow{z^{0}}$. a perturbative Hamiltonian $H^{\prime}=-\vec{r} \cdot \vec{E}$ is produced. which is $\mathrm{P}$ - and $\mathrm{CP}$-even. The corresponding first-order EM energy is

$$
E^{\prime}(\alpha)=\{2 \cdot(\alpha)|-e \vec{r} \cdot \vec{E}| 2 \cdot(\alpha)\} .
$$

and one finds an $\alpha$-dependence of $E^{\prime}(\alpha)$ because $H^{\prime}$ is not $O(4)$-invariant. Of course. the energy of $\mathrm{H}$-atom in external fields should still be two-valued instead of being a continuous function of $\alpha$. Something is clearly wrong. Lsing the diagonalization method to get things straight. one finds the eigenvalues of $H^{\prime}$ to be $E_{ \pm}^{\prime}=\mp 36 a E_{z}\left(a=\epsilon^{2} / 4 \pi \mu\right.$. and $\mu$ is the 
reduced mass), and the corresponding wave functions:

$$
|\psi \pm\rangle=1 / \sqrt{2}(|1\rangle \pm|2\rangle)
$$

(The other two $n=2$ wave functions are not affected. nor are their energies.) These states can also be found by taking variation. i.e., taking the solutions of the following equation:

$$
\frac{\partial}{\partial \alpha} E^{\prime}(\alpha)=0
$$

In this example, the two states in Eq. (3) are neither eigenstates of orbital angular momentum nor those of parity. The perturbative energy, however, is still P-even.

In more general cases, the original symmetry of the Lagrangian may not be realized in the physical results. When the fermion masses are allowed to have chiral phases (as in the standard model of the electro-weak interactions), the parity-odd solutions often creep in undetected and seem reasonable as the mass term breaks standard P- and T-symmetry. Therefore, the study of the degeneracy is very appealing.

The related problem does not exist if one does not start dynamical calculations with solutions of the free Dirac equation. such as in obtaining the spectrum of the Dirac equation with the Coulomb potential (DECP). For this reason it is possible to solve the problem of how to choose the lowest-order wave functions by taking a hint from the DECP solutions. We shall do so and explain why. As a by-product of this study. we obtain some interesting sum rules for pure Coulomb-potential wave functions.

Since the degeneracy is very similar for the masses with and without a chiral phase. we shall first study the standard QED in which fermion mass is real. We shall then study the QED theory in which fermion mass has a chiral phase, or is complex. as we say.

\subsection{DIRAC EQUATION WITH REAL FERMION MASS}

For convenience, we use the Bjorken-Drell convention ${ }^{3}$ of the Dirac matrix and spacetime metric. The free Dirac equation is

$$
\not D u=\left(\begin{array}{cc}
E-m & \cdot \vec{\sigma} \cdot \vec{p} \\
\vec{\sigma} \cdot \vec{p} & E+m
\end{array}\right)\left(\begin{array}{l}
\hat{\tau} \\
\chi
\end{array}\right)=0 .
$$

Ont obtains a naive solution:

$$
u_{+}=\sqrt{\frac{E+r n}{2 E}}\left(\begin{array}{c}
\hat{\tau} \\
\frac{\vec{\sigma} \cdot \vec{p}}{I_{-}+m} \gamma
\end{array}\right) .
$$


where $\varphi$ is a two-component Pauli spinor. With the same $\varphi$,

$$
u_{-}=\sqrt{\frac{E-m}{2 E}}\left(\begin{array}{c}
\frac{\vec{\sigma} \cdot \vec{p}}{E-m} \varphi \\
\varphi
\end{array}\right)
$$

is another solution of the free Dirac equation. Under space reflection $\psi(\vec{p}) \longrightarrow \gamma_{0} \psi(-\vec{p})$, $u_{+}$and $u_{-}$are, respectively, parity-even and -odd, if $\varphi$ is parity-even (and we shall assume so). Any linear combinations of $u_{+}$and $u_{-}$are also the solutions of the Dirac equation with the same energy and momentum. It is convenient to parametrize part of these degenerate solutions in the following way. (For the general expression, see Appendix A.) Let

$$
U(\beta)=[\cos \beta+i \vec{\Sigma} \cdot \vec{p} \sin \beta /(E+m)] / \sqrt{E+m \cos 2 \beta},
$$

where $\vec{\Sigma}=\left(\begin{array}{cc}\vec{\sigma} & 0 \\ 0 & \vec{\sigma}\end{array}\right)$ and $\beta$ is an arbitrary real parameter. Then

$$
\begin{aligned}
\tilde{u}(\beta) & =\mathcal{L}(\beta) u_{+} \\
& =\frac{1}{\sqrt{2 E(E+m \cos 2 \beta)}}\left[\left(\begin{array}{c}
E+m \\
\vec{\sigma} \cdot \vec{p}
\end{array}\right) \cos \beta+\left(\begin{array}{c}
\vec{\sigma} \cdot \vec{p} \\
E-m
\end{array}\right) i \sin \beta\right]
\end{aligned}
$$

is also the solution of the Dirac equation with the same 4-momentum. This can be understood in a different way. $U(3)$ is a unitary transformation which produces one solution from another, since $l^{*}(\beta)$ commutes with the Dirac operator,

$$
\not D L(\beta)=U(\beta) \not D
$$

The set $\mathcal{L}(\beta)$ is a degenerate group of the Dirac equation. Since $\vec{\Sigma}$ is a block diagonalized matrix. $\tilde{u}(3)$ describes a pure electron (without the component of the positron) as $u_{+}$does. Of course, all these wave functions are equally good in describing a free electron. Now, an immediate question is whether perturbative calculations with these different solutions as lowest-order wave functions give the same result when interactions are imposed. The problem is of the type of perturbative theory with degenerate wave functions, and the answer is obviously not.

When perturbative external fields are added to the Dirac equation. the Hamiltonian operator will have an extra part $\dot{H}^{\prime}$ in addition to the free Dirac Hamiltonian 
$\hat{H}_{o}=\vec{\alpha} \cdot \vec{p}+\gamma_{o} m$. In general,

$$
\left[U(\beta), \hat{H}^{\prime}\right] \neq 0
$$

Therefore.

$$
E^{\prime}(\beta)=\left\langle\epsilon\left|\psi^{\dagger} \hat{H}^{\prime} \psi\right| \epsilon\right\rangle=\tilde{u}^{\dagger}(3) \hat{H}^{\prime} \tilde{u}(\beta)
$$

will be $\beta$-dependent, where $\tilde{u}(\beta)$ is the identified wave function of the free electron, as all the wave functions have an equal right to be considered. The $\beta$-dependence of the firstorder energy in Eq. (11) is quite embarrassing. It seems that the two methods to solve the problem of the H-atom are not practically useful here. To see the gravity of the problem raised, let us calculate the first-order electromagnetic energy of the electron in external EM fields explicitly. Suppose scientist A chooses $u_{+}$of Eq. (5) as the free wave function of the electron; he obtains

$$
E_{A}^{\prime}=\varphi^{\dagger}\left[e \Phi-\frac{e \vec{A} \cdot \vec{p}}{E}-\frac{e \vec{\sigma} \cdot \vec{B}}{2 E}-\frac{e \vec{\sigma} \cdot \vec{E} \times \vec{p}}{2 E(E+m)}+\frac{i e \vec{E} \cdot \vec{p}}{2 E(E+m)}\right] \varphi .
$$

This two-component form is better than Eq. (11) in the sense that physical contents such as the E.M potential, the magnetic moment, and the spin-orbit interaction (the 4th term) are explicitly seen. He also obtains the EM energy of the positron with the corresponding wave function $\sqrt{\frac{E+m}{2 E}}\left(-\varphi^{\dagger} \frac{\vec{\sigma} \cdot \vec{p}}{E+m} \varphi^{\dagger}\right)$ of exactly $-E_{A}^{\prime}$. But scientist B may choose a different solution- $-\tilde{u}(\beta)$. for example--to start with. Not knowing what $\mathrm{A}$ is doing, $\mathrm{B}$ finds that

$$
E_{B}^{\prime}=\varphi^{\dagger}\left[\epsilon \Phi-\frac{\epsilon \vec{A} \cdot \vec{p}}{E}-\frac{\epsilon \vec{\sigma} \cdot \vec{B}}{2 E}-\frac{\epsilon \vec{\sigma} \cdot \vec{E} \times \vec{p}}{2 E\left(E+m_{1}\right)}+\frac{i e \vec{E} \cdot \vec{p}}{2 E\left(E+m_{1}\right)}-\frac{\epsilon m_{2} \vec{\sigma} \cdot \vec{E}}{2 E\left(E+m_{1}\right)}\right] \hat{\psi} .
$$

with $m_{1}=m \cos 23$ and $m_{2}=m \sin 23$. His positron wave function will be

$$
\frac{1}{\sqrt{2 E(E+m \cos 2,3)}}\left[\left(\begin{array}{c}
-\vec{\sigma} \cdot \vec{p} \\
E+m
\end{array}\right) \cos \beta+\left(\begin{array}{c}
E-m \\
-\vec{\sigma} \cdot \vec{p}
\end{array}\right) i \sin \beta\right] .
$$

with the energy $-E_{B}^{\prime}$. Similar differences appear in second-and higher-order calculations. The main characteristic of $E_{B}^{\prime}$ is that there is an EDM term in it, with an EDM equal to $\epsilon \tan 3 / 2 m$ in the non-relativistic limit. (There are also other differences between $E_{A}^{\prime}$ and $E_{B}^{\prime}$.) Scientist A may argue that since the original Lagrangian is parity-conserved. there should be no parity-violating effect such as EDM in the result. However, B may contradict $A$ by saying that sometimes the symmetry of the Lagrangian may not be preserved in dynamical calculations-for exanple. when dynamical symmetry hreakdown occurs. Therefore. further arguments should be giver before any conclusions are reached. 
A lesson from the above exercise is that people working in different representations of the $\gamma$-matrices tend to obtain different perturbative results if special care is not taken. This is simply because they may get different results even if they work in the same representation by accidentally taking different lowest-order solutions (see Appendix A). It is interesting to note that if one somehow succeeds in rejecting EDM in Eq. (13), one will obtain $\sin 2 \beta=0$, which allows

$$
\cos 2 \beta= \pm 1
$$

The second solution $(\cos 2 \beta=-1)$ leads to a divergent $E_{B}^{\prime}$ in the non-relativistic limit, making it an unfavorable one. We shall give the necessary argument to reject EDM later.

It seems to us that these uncertainties cannot be eliminated unless a hint from the nonperturbative solutions of an interacting Dirac equation is applied. Actually, the solutions of the Dirac equation with the Coulomb potential are good enough to be compared with, because they are exact and can be made into an orthogonal series. The corresponding equation is

$$
\left(\vec{p} \cdot \vec{\alpha}+\gamma_{o} m-z e^{2} / r\right) \psi=E \psi
$$

The exact solutions of this equation are known. ${ }^{4}$ They are characterized by the quantum numbers $\left(n, j, l, j_{z}\right)$ which are, respectively, the principal, total angular momentum, orbital angular momentum, and magnetic quantum numbers. In addition to the degeneracy of degree $2 j+1$. one more degeneracy appears between states with the same $n$ and $j$ but different $l$, unless $l=0$ or $j=n-1 / 2$. These degenerate states have different parities. There are no parity-violating terms in the energy even if we consider a parity-mixed state, because the Coulomb potential is spherically symmetric. We expect the value of $\vec{\sigma} \cdot \vec{E}$ in the pure Coulomb wave functions to vanish anyway. So to see whether the electron has an EDM. we must add an external electric field $H^{\prime}=-\vec{E} \cdot \vec{r}$ as a perturbation to the Coulomb potential solutions. This perturbation will certainly split the degeneracy between states with different parities as previously discussed. Had the electron had an EDM, we would also expect a splitting among states with different $j_{z}$ but with the same other quantum numbers, in particular for states with $l=0$ and $j_{z}=s_{z}$. However, it is easy to see that this kind of splitting does not appear, leading to the conclusion that the electron does not have any EDM. Therefore, we must take the free solution $u_{+}$as the wave function of a free electron to start perturbation calculations in the real mass situation, if we believe that EDM should not be produced only because the electron goes from the Coulomb potential to some other arbitrary potentials and perturbation based on free Dirac equation solutions 
must apply. The problem of which free solution should be taken for perturbation for the real mass case is thus resolved.

\subsection{DIRAC EQUATION WITH COMPLEX FERMION MASS}

Similar problems appear in the perturbative QED with a complex fermion mass (in other vords, the fermion mass with a chiral phase). The complex fermion mass $\tilde{m}$ is defined as $\tilde{m}=m \epsilon^{-i \gamma_{5} \phi}=m \cos \phi-i \gamma_{5} m \sin \phi \equiv m_{1}-i_{\gamma_{5}} m_{2}$. The Lagrangian is

$$
\mathcal{L}=-1 / 4 F^{\mu \nu} F_{\mu \nu}+i \bar{\psi}(\not \partial+i e . A) \psi^{\prime}-\bar{\psi} \tilde{m} \psi
$$

It seems that the Lagrangian of Eq. (16) breaks $\mathrm{P}$ and $\mathrm{CP}$, but leaves $\mathrm{C}$ conserved. Researchers sometimes make chiral rotation to shift this phase of the mass into the EM part using the anomaly relations, ${ }^{5}$ then throw it away. We shall instead work on $\mathrm{Eq}$. (16) directly. At the very least. everybody knows that chiral rotation is not the symmetry of the Lagrangian. A double check will not cause any harm.

The perturbation theory based on $\mathcal{L}$ in Eq. (16) is as good as that in the standard QED. "The question again is which solution of the "free" Dirac equation

$$
(\not p-\tilde{m}) \psi=0
$$

should be taken as the lowest-order solution to start perturbation. The general solutions of Eq. (17) may be written as

$$
\tilde{u}_{c}(\beta)=e^{i \gamma_{s} \phi / 2} \tilde{u}(\beta)
$$

where $\tilde{u}(3)$ with an artitrary $\beta$ is the solution of the real-mass eonuion in Eq. (8). ()ne possible method to choose a specific solution is to think about the o-goes-to-zero limit to establish a relation between the chosen real-mass solution and the complex-mass solution. For example. if $u+$ in Eq. (5) is chosen for the real-mass equation. then $\tilde{u}_{c}(3)$ with $: 3=6,2$ or 0 only sheuld be chosen for the complex-mass equation.

$$
u_{c}(1)=\ddot{u}_{c}(\xi=0)=\epsilon^{1 ; \vdots 0 / 2} u_{+}
$$

aId

$$
u_{c}(2)=\tilde{u}_{c}(j=0 / 2) \times\left(\begin{array}{c}
\vec{T} \\
\frac{\vec{\sigma} \cdot \vec{p}+t m_{2}}{E+m}+m_{2}
\end{array}\right) .
$$

Both of these solutions approach the real-mass solution $u+$ at the limit $0-0$. and we

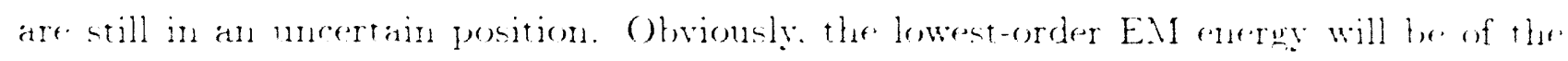

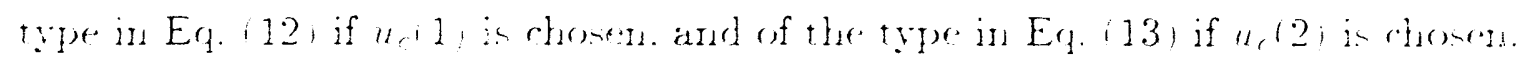


Appealing to the corresponding Coulomb potential solutions, we find that

$$
\left(\vec{p} \cdot \vec{\alpha}+\gamma_{o} \tilde{m}-z \epsilon^{2} / r\right) \psi_{c}=\tilde{E} \psi_{c} .
$$

If $\psi_{c}^{\prime}=\epsilon^{i \gamma_{5} \phi /{ }^{\prime}} \iota_{c}$, we find that $\psi_{c}^{\prime}$ satisfies exactly the same equation with the same energy $\tilde{E}=E$ as $\psi$ in $\mathrm{Eq}$. (15) does. Therefore, we have

$$
\psi_{c}^{\prime}=\text { a mixture of } \psi(1) \text { and } \psi(2)
$$

where $\psi(1)$ and $\psi(2)$ are degenerate solutions of Eq. (15) with the same total energy E and angular momentum $\left(j, j_{z}\right)$, but different parity as $l=j \pm 1$. So the energy of $\psi_{c}$ is the same as $\psi_{c}^{\prime \prime}$, or $\psi(1)$ and $\psi^{\prime}(2)$, and it has no P-odd pieces even though it is the solution of the Dirac equation with a complex fermion mass. This means that we must take the perturbative $\epsilon_{n}$ ergy of the eleciron in Eq. (12) instead of Eq. (13), and we must take the corresponding free-electron wave function in Eq. (19) instead of Eq. (20) to do perturbation in QED with a complex fermion mass.

\subsection{CONCLUSION}

In conclusion, we must take the parity-even solution in Eq. (5) to start perturbation calculation if the mass of the fermion is real. When the mass of the fermion is complex, we must take the solution in Eq. (19) to start perturbation. so the phase of the complex fermion mass becomes a completely redundant parameter without any physical consequence in QED. The non-observability of the phase of the complex mass in QED can be used to find some interesting sum rules for wave functions (Appendix B). Implications of the results of this paper to the EDM of the neutron should be discussed separately, as dynamical symmetry breakdown due to the strong interaction should be taken into account. ${ }^{7}$ 


\section{REFERENCES}

1. H. Banerjee, D. Chatterjee, and P. Mitra. Saha Institute preprint, SINP-TNP-90/こ and 90/17: R.G. Sachs, Enrico Fermi Institute preprint: A. Abada. J. Galand, A. Le Yaouanc, L. Oliver, O. Pene, Phys. Lett. 256B, 508 (1991). In contrast to the previous works: R. Peccei and H. Quin, Phys. Rev. Lett. 38, 1440 (1976): Phys. Rev. D16, 1791 (1977); V. Baluni, Phys. Rev. D19, 2227 (1979). M.A. Shifman, A.I. Vainshtein, and V.I. Zakharov, Nucl. Phys. B166, 493 (1980); R.J. Crewther. P.Di Vecchia, G. Veneziano, and E. Witten, Phys. Lett. 88B, 123 (1979).

2. See L.I. Schiff, Quantum Mechanics, McGraw-Hill, 1949, p. 157.

3. J.D. Bjorken and S.D. Drell, Relativistic Quantum Fields, McGraw-Hill, 1964, p. 281.

4. C.G. Darwin. Proc. Roy. Soc. (London), A118, 654 (1928); W. Gordon, Z. Physik, 48. $11(1928)$.

5. J. Steinberger. Phys. Rev. 76, 1180 (1949); S. Adler, Phys. Rev. 177, 2426 (1969); J. Bell and R. Jackiw. Nov. Cim. 60, 47 (1969); W.A. Bardeen, Phys. Rev. 284, 1848 (1969); J. Wess and B. Zumino, Phys. Lett. 37B, 95 (1971); K. Fujikawa, Phys. Rev. Lett. 42. 1195 (1979) and Phys. Rev. D21, 2848 (1980).

6. B.H.I. McKellar and D.D. Wu. Melbourne University preprint.

7. Z. Huang. K. Viswanathan, and D.D. Wu, Simon Fraser University preprint, July-90-1. unpublished: and Mod. Phys. Lett. A 8, 711 (1991). 


\section{Appendix A}

\section{A Comparison of the Bjorken-Drell Representation and the Chiral Representation}

The chiral representation is defined as

$$
\dot{\gamma}_{i}=\left(\begin{array}{cc}
0 & \sigma_{i} \\
-\sigma_{i} & 0
\end{array}\right), \quad \dot{\gamma}_{o}=\left(\begin{array}{cc}
0 & I \\
I & 0
\end{array}\right), \quad \dot{\gamma}_{5}=\left(\begin{array}{cc}
-I & 0 \\
0 & I
\end{array}\right) .
$$

The unitary matrix $V=1 / \sqrt{2}\left(\begin{array}{cc}I & I \\ -I & I\end{array}\right)$ transforms it back into the B-D representation:

$$
V \dot{\gamma}_{n} V^{-1}=\gamma_{n}, \quad n=0,1,2,3,5
$$

The "naive" solution of the free Dirac equation $(\not \not-m) \dot{u}=0$ is

$$
\dot{u}=\frac{m^{2}}{\sqrt{2 E(E+h p)}}\left(\begin{array}{c}
\eta \\
\frac{E+\vec{\sigma} \cdot \vec{p}}{m} \eta
\end{array}\right)
$$

where $h$ is the helicity of the two-component spinor $\eta$. Probably the author working in the chiral representation will identify $\eta$ as a momentum-independent spinor. It is obvious that

$$
V \dot{u} \equiv u^{\prime}=\frac{m^{2}}{2 \sqrt{E(E+h p)}}\left(\begin{array}{c}
\frac{E+m+\vec{\sigma} \cdot \vec{p}}{m} \eta \\
\frac{E-m+\vec{\sigma} \cdot \vec{p}}{m} \eta
\end{array}\right) \neq u
$$

where $u$ is the naive solution in the B-D representation. So with $\dot{u}$ and $e \dot{A}$, and using Eq. (11), this author obtains his first-order EM energy:

$$
\dot{E}^{\prime}=\dot{u}^{\dagger} \dot{\gamma}_{o} \epsilon \dot{A} \dot{u}=\eta^{\dagger}\left[e \Phi-\frac{\epsilon \vec{A} \cdot \vec{p}}{E}-\frac{e \vec{\sigma} \cdot \vec{B}}{2 E}+\frac{i e \vec{\sigma} \cdot \vec{E}}{2 E}\right] \eta
$$

The same result is obtained by the use of $u^{\prime}$ and $\epsilon \not A$. Note that this energy is not even one of those in Eq. (13), because Eq. (8) does not represent all possible degenerate wave functions. The most general expression for the solvtions of the free Dirac equation in the $B-D$ representation is

$$
\tilde{u}=(A+B \vec{\Sigma} \cdot \ddot{\jmath}) u_{+}
$$

with complex numbers $\mathrm{A}$ and $\mathrm{B}$ constrained by $\tilde{u}^{\dagger} \tilde{u}=1$. 


\section{Appendix B}

\section{The Sum Rules Obtained From "No Effect" of the Complex Fermion Mass}

When the phase $\phi$ is small, we can write

$$
\tilde{m}=n^{\prime}+\tilde{m}^{\prime}
$$

where the small quantity $\tilde{n}^{\prime}$ is

$$
\tilde{m}^{\prime}=-m(1-\cos \phi)-i m \gamma_{5} \sin \phi
$$

The Dirac equation with $a$ complex fernion mass becomes

$$
\left[\vec{\alpha} \cdot \vec{p}+\gamma_{o} m-e^{2} / r+\tilde{m}^{\prime}\right] \psi=\left(E_{o}+\delta E\right) \psi
$$

whre $E_{o}$ is tire energy corresponding to $H_{0}$ (when $\tilde{m}^{\prime}=0$ ), anu $\delta E$ is the energy due to $\bar{m}^{\prime}$. Since all lowest order wave functions are choser to be eigenfunctions of parity, $\gamma_{5}$ must appear twice to give non-zero contribution. Therefore, $\delta E$ can only be an even function of $\phi$. From the text we expect that we must have $\delta E=0$. This is not true for each order of perturbative calculations, but it, must somehow be satisfied. Actually we can require the coefficient of the $\phi^{2 n}$ term to vanish, where $n$ is a positive integer. This requirement supplies an infinite number of sum rules for Coulomb potential solutions. As an example, the $0^{2}$ terms come from the first-and second-order perturbations, and in order to have these terms cancel each other, we must have

$$
\left\langle\psi_{0}\left|\gamma_{0}\right| \psi_{o}\right\rangle=-m \sum^{\prime} \frac{\left\langle\psi_{o}\left|\gamma_{0} \gamma_{5}\right| n\right\rangle\left\langle n\left|\gamma_{0} \gamma_{5}\right| \psi_{o}\right\rangle}{E_{o}-E_{n}}
$$

where $\%$ has it energy $E_{o}$, and the sum must skip the intermediate state $|n\rangle$ with energy $E_{0}$. This sum rule is directly proved if one notices that

$$
\gamma_{10} i_{5}=1 / 2 m\left[H_{0}, \gamma_{5}\right] \text {. }
$$



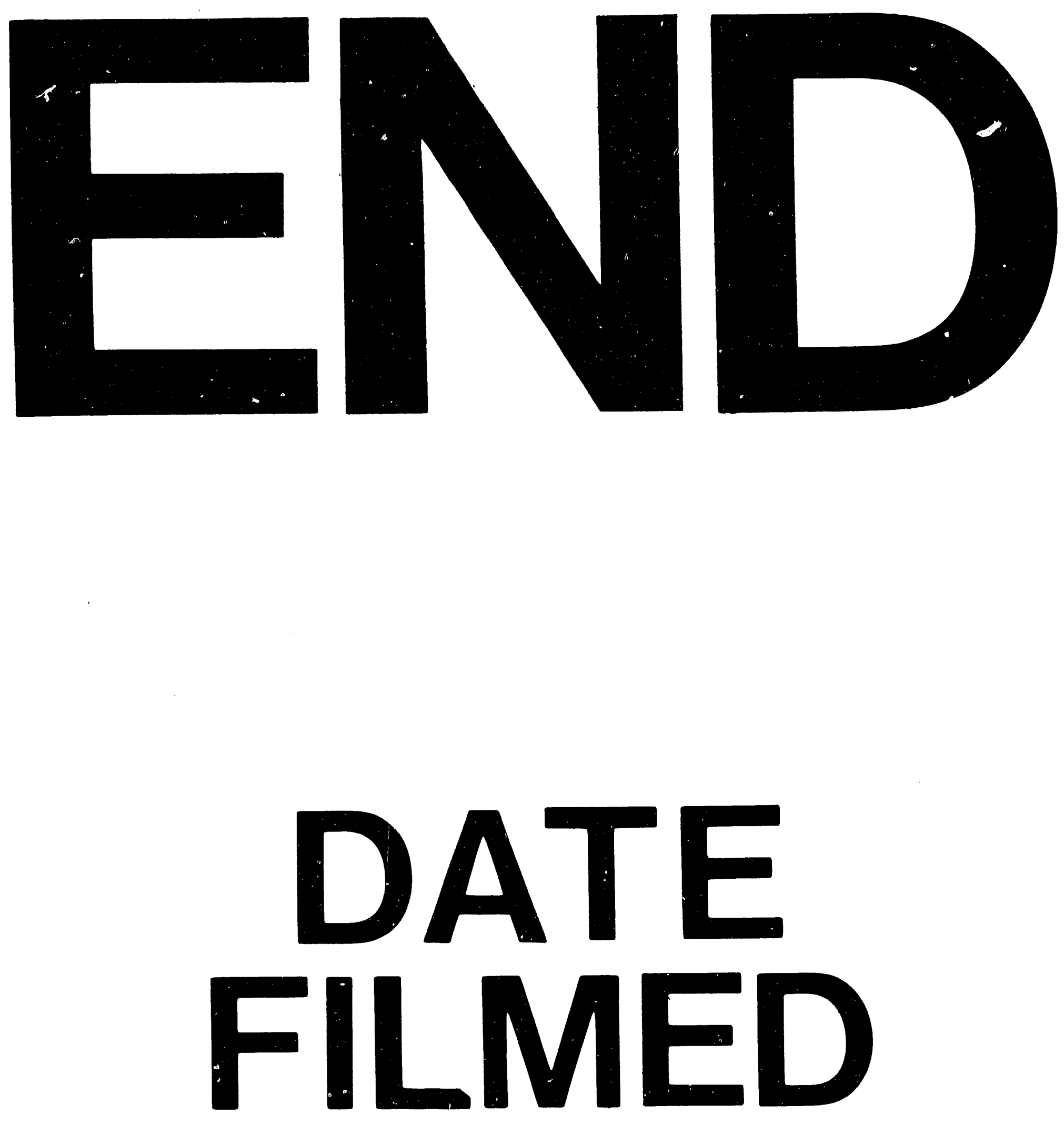

1

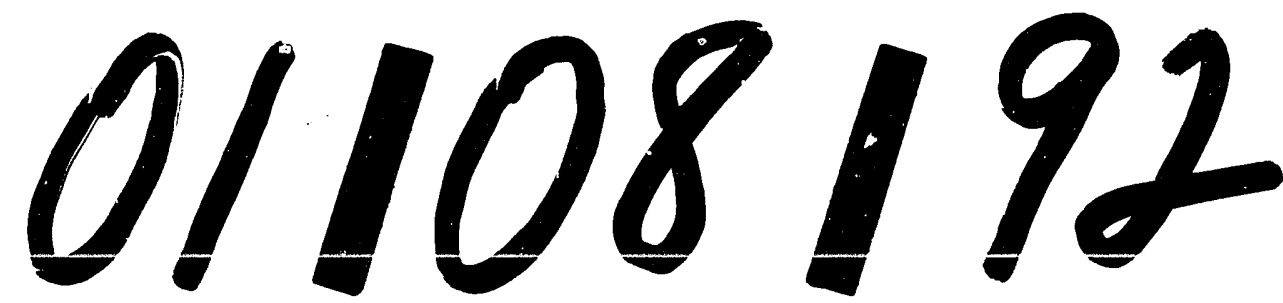


